Memorias del VII Encuentro Nacional de Experiencias en la Enseñanza de la

Biología y la Educación Ambiental y II Congreso Nacional de Investigación en la Enseñanza de la Biología

\title{
FORMACIÓN DE PROFESORES DEL DISTRITO CAPITAL EN EDUCACIÓN AMBIENTAL DESDE LA PERSPECTIVA INTERDISCIPLINAR. PROBLEMA DE INVESTIGACIÓN ${ }^{1}$
}

\author{
Jenny Johanna Duarte Díaz ${ }^{2}$ \\ Édgar Orlay Valbuena Ussa ${ }^{3}$
}

\section{Resumen}

Uno de los retos que se le ha impuesto a la educación actual está dado en la educación ambiental, por lo que las naciones han modificado sus políticas incluyendo la presencia de la dimensión ambiental y la formación en educación ambiental desde la escuela. Sin embargo a pesar del importante rol que desempeñan los profesores de todas las disciplinas en los procesos de enseñanza aprendizaje, se tiene poco en cuenta su formación en este ámbito, dejando la tarea de la educación ambiental como un campo exclusivo de los docentes de Ciencias Naturales y particularmente los licenciados en biología.

Desde allí es necesario proponer ambiciosos proyectos de investigación que tengan como objetivo conseguir la formación de docentes de diferentes áreas del conocimiento en educación ambiental, abarcando a los profesores en formación y a los que ya se encuentran en ejercicio. Partiendo de la identificación de sus contextos específicos y caracterizando sus propias representaciones sociales de educación ambiental, para finalmente diseñar e implementar estrategias conjuntas que logren la transformación del discurso en las comunidades educativas que integran. Esta ponencia presenta elementos de la formulación del problema de investigación correspondiente a la de tesis doctoral de uno de los autores.

\section{Abstract}

One of the challenges imposed to the current education is closely related with environmental education, that's why nations has been modifying their policies including the environmental dimension and the formation in environmental education from the school. However, despite the important role that teachers of all disciplines has on the process of teaching and learning processes in this area, it is being sub estimated, leaving the environment education as an exclusive field for natural sciences teachers and particularly for biology graduates.

\footnotetext{
${ }^{1}$ Esta ponencia hace parte de la formulación del proyecto de tesis doctoral, el cual se desarrollará en el Grupo de Investigación Conocimiento Profesional del Profesor de Ciencias.

${ }^{2}$ Estudiante Doctorado Internacional en Educación. Docente Secretaria de Educación Distrital.

${ }^{3}$ Doctor en Didáctica de las Ciencias Experimentales. Docente Universidad Pedagógica Nacional.
} 
Bio-grafia Escritos sobre la Biologia y su Enseñanza.

Edición Extra-Ordinaria. ISSN 2027-1034 P. p196 - 204

Memorias del VII Encuentro Nacional de Experiencias en la Enseñanza de la

Biología y la Educación Ambiental y II Congreso Nacional de Investigación en la Enseñanza de la Biología

From there is necessary to proposals ambitious research projects that involves as an objective get the training of teachers from different areas of knowledge in environmental education, including teachers in formation and already in exercise.Starting from the identification of their specific contexts and characterizing their own social representations of environment education, to finally design and apply joint strategies to achieve the transformation of the speech in the educational communities that make up.

In this paper we present elements of the research problem related to the doctoral dissertation of one of the authors.

Palabras clave: Formación profesional del profesor, ambiente, educación ambiental, instituciones educativas.

Keywords:_training teacher, environment, environmental education, educational institutions.

\section{Introducción}

De los grandes desafíos de la educación en los últimos años se puede reconocer el que está dado en el campo de la Educación Ambiental, como lo plantea Sauvé (2002) la tarea es inmensa y compleja, por lo que es importante ubicar a los maestros como agentes activos en los procesos de enseñanza- aprendizaje capaces de intervenir en los diferentes contextos y lograr una transformación efectiva en las visiones que poseen las comunidades que integran. Reconociendo que en la actualidad generalmente en las instituciones educativas no se construyen de manera consciente los procesos de la educación ambiental, debido a que las prácticas pedagógicas tradicionales no permiten la incorporación de ésta dimensión en la escuela porque como lo planteaba González (2000) aún se mantiene una época de resistencia en la que se piensa que el ambiente ya forma parte del contenido escolar porque se habla de la vegetación y fauna, sin reflexionar frente a que el ambiente no tiene como única concepción la natural.

Se propone así, un problema de investigación desde el conocimiento profesional del profesor que aporte a la formación de docentes, en aras de transformar la perspectiva reduccionista de ambiente y educación ambiental en la escuela. Desde un abordaje interdisciplinar, para contribuir a la construcción de una nueva cultura en comunidades educativas, en la cual se incluya al ser humano como constituyente fundamental del ambiente. 
Bio-grafia Escritos sobre la Biologia y su Enseñanza.

Edición Extra-Ordinaria. ISSN 2027-1034 P. p196 - 204

Memorias del VII Encuentro Nacional de Experiencias en la Enseñanza de la

Biología y la Educación Ambiental y II Congreso Nacional de Investigación en la Enseñanza de la Biología

\section{Metodología}

Para la formulación de esta problemática de investigación la postura que se ha venido direccionando ha sido dada desde el paradigma interpretativo referido a la "teoría que constituye una reflexión y desde la praxis conforma la realidad de hechos, por significados elaboradas del propio sujeto" (Cohen y Manion 1990, p.18).Abordada desde un enfoque cualitativo en el que se propone que quien investiga construya una imagen compleja y holística, analizando las palabras y presentando detalladas perspectivas de los informantes.(Creswell 2006 en Vasilachis 1998 p.2).

Se ha avanzado en la revisión documental y la contextualización de los espacios a trabajar identificando que cada una delas localidades del Distrito Capital presenta una realidad y una dinámica particular, por lo cual para la formulación de la investigación se han referenciado las descripciones específicas que se han adelantado desde los Planes de Ordenamiento Territorial (POT 2012-1016) y los diagnósticos de aspectos físicos, demográficos y socioeconómicos que realizó la Secretaria Distrital de Planeación durante el año 2009. Para de esta manera contribuir en el diseño de estrategias de formación en educación ambiental que se encuentren acordes con la situación de cada localidad.

Es importante resaltar que en los objetivos específicos del actual plan de ordenamiento territorial para el Distrito Capital se propone como una de las estrategias, "desarrollar procesos pedagógicos y de formación de mediano y largo plazo, que modelen una nueva ética ambiental y hagan de Bogotá un aula que reconozca y revalorice los diferentes territorios ambientales" (POT, 2012 p. 191). Para lo que la Secretaria de Educación Distrital ha establecido el trabajo en las instituciones educativas con procesos de educación ambiental que hagan parte del Proyecto de Educación para la Ciudadanía y la Convivencia (PECC), a través del cual se pretende promover acciones pedagógicas que permitan proponer proyectos educativos que incidan, no solo en el nivel institucional, sino también en la transformación de las realidades ambientales de su entorno (SED, 2013).

En este contexto resulta viable fortalecer la formación de los maestros de diferentes disciplinas pertenecientes al Distrito Capital, que estén dispuestos a construir procesos y transformar su discurso ambiental, pensándolo desde una visión holística que permita identificar al ser humano dentro del entorno.

\section{Resultados}

Un primer paso para la transformación de las visiones de ambiente, es reconocer planteamientos de autores como L. Sauvé, que desde los años 90ha venido proponiendo tipologías de los diferentes conceptos de ambiente, convirtiéndose en un gran referente para estudios posteriores en el tema. Esta autora ha presentado 
Bio-grafia Escritos sobre la Biologia y su Enseñanza.

Edición Extra-Ordinaria. ISSN 2027-1034 P. p196-204

Memorias del VII Encuentro Nacional de Experiencias en la Enseñanza de la

Biología y la Educación Ambiental y II Congreso Nacional de Investigación en la Enseñanza de la Biología

diversos tipos de conceptos, de los que podemos destacar en el ámbito escolar han sobresalido "el ambiente como recurso para administrar", muy usual en la actualidad por estar directamente relacionado a la calidad de vida en donde se percibe que el ambiente se agota y se degrada, por lo que se debe aprender a administrar para conseguir un desarrollo sostenible. Concepción en la cual podría pensarse, se ubican las políticas de educación actuales, que proponen la educación ambiental como un instrumento que contribuya a mejorar las condiciones de vida de los seres humanos (Decreto 617 de 2007). Otra tipología importante desde la escuela hace referencia "al ambiente como naturaleza" en la que se propone un vínculo directo con los seres vivos y sus interacciones. La cual es trabajada en las instituciones por su inclusión en el currículo de ciencias naturales, específicamente en el componente ecosistémico de la disciplina biológica. Y finalmente el concepto de "ambiente como medio de vida para conocer y administrar" presentado como uno de los más reconocidos en algunos contextos escolares del distrito capital (Duarte et al., 2013; Pérez et al.,2007) por que se vincula a los entornos cotidianos del ser humano: escolar, familiar y laboral.

Estas tipologías proponen la manera de definir las prácticas específicas de los grupos que integran una comunidad, lo que puede permitir que representadas en su conjunto contribuyan a asumir una postura de ambiente desde una mirada global que implique las relaciones con el espacio natural y social, en momentos históricos particulares, reconociendo las características contextuales y valorando la pluralidad de pensamiento, con el fin de direccionar la construcción de ideas que partan de la reflexión colectiva. Idea que coincide con la visión de ambiente propuesta por Pulido (2002, citado en Jessup et al., 2010) quien lo expone como un "sistema dinámico en donde se establecen interrelaciones entre factores físicos, biológicos, sociales y culturales, en las cuales se expresan tanto las diferentes condiciones de la naturaleza como las propias de lo humano, pues las personas forman parte del mismo" (p. 37).

No obstante no resulta fácil esta transformación, también es necesario reconocer el contexto por el que ha venido atravesando la educación ambiental. En términos generales para Latinoamérica se presentan sus primeros pasos en los años ochenta con la idea de que existe una crisis ambiental, "resultado de la crisis civilizatoria occidental, causada por sus formas de conocer, concebir, y por ende transformar, el mundo" (Eschenhagen, 2008 p.2) a partir de lo cual en cada país se inicia la construcción de propuestas de implementación en educación ambiental, buscando estimular los procesos formativos de la escuela. Así, a nivel nacional en la Constitución Política de 1991, en varios de sus artículos (Art. 8, 58, 79 y 95) se incorporan los derechos ambientales señalando la importancia del papel de la educación ambiental en la transformación de la sociedad y en los procesos de desarrollo sostenible. Adicional desde el Ministerio de Educación (Decreto 1743, 1994) y la Ley general de Educación (1994) se dirección a la 
Bio-grafia Escritos sobre la Biologia y su Enseñanza.

Edición Extra-Ordinaria. ISSN 2027-1034 P. p196 - 204

Memorias del VII Encuentro Nacional de Experiencias en la Enseñanza de la

Biología y la Educación Ambiental y II Congreso Nacional de Investigación en la

\section{Enseñanza de la Biología}

obligatoriedad de la educación ambiental en las instituciones educativas y la implementación de proyectos ambientales escolares (PRAES), que busquen solucionar problemáticas ambientales del contexto. Y a nivel específico en el Distrito Capital de Bogotá, a partir del Decreto 617 de 2007, la educación ambiental es considerada "un instrumento para la construcción de una ética ambiental que debe coadyuvar a la mejora de las condiciones de vida de quienes habitan en el distrito capital, a partir del reconocimiento de procesos y relaciones territoriales históricas, simbólicas y socioculturales, desde las cuales, se contextualizan las situaciones ambientales conflictivas y las acciones pertinentes para su abordaje" (Colombia Aprende, 2002).

Sin embargo esta linealidad desde el ámbito internacional que tiene como finalidad la aplicación de las políticas generales de la educación ambiental en los contextos particulares, salta abruptamente desde lo Distrital a las instituciones educativas no construyendo directrices locales y generando que la educación ambiental no logre articularse de forma apropiada con las estructuras y prácticas, ya que las recomendaciones derivadas de diagnósticos generales no se adoptan a problemáticas específicas. Es decir desde la política distrital no existe una conexión directa con las entidades locales que manejan las mesas ambientales y mucho menos una vinculación con las instituciones de educación superior en las que se forman los licenciados de las diversas disciplinas, por lo que no se construyen planteamientos colectivos que direccionen los trabajos en las escuelas vinculando sus problemáticas al contexto en el que se encuentran.

Particularmente en los últimos años lo que ha venido ocurriendo en las instituciones educativas es que algunas entidades (Universidad Autónoma 2011; Universidad Pedagógica Nacional 2013) en cortos convenios con la Secretaría de Educación Distrital vinculan a pequeños grupos de docentes del área de ciencias naturales y unos pocos estudiantes para adelantar procesos de conformación de comités y específicamente generar actividades en busca de la sensibilización ambiental temporal, no dejando claro que la educación ambiental no se puede centrar en la realización de actividades que busquen garantizar recursos materiales para el bienestar del ser humano.

Por lo cual la problemática real de las comunidades educativas se establece en la carencia de un trabajo organizado desde una perspectiva interdisciplinaria que permita dentro del conocimiento profesional del profesor, establecer conexiones entre las diferentes disciplinas y campos del conocimiento con la realidad, propiciando un enfoque integrador que problematice la interacción entre la sociedad y la naturaleza (Teixeira et al., 2013). Entendiendo la interdisciplinaridad ambiental, no como la adición de asignaturas a la educación ambiental, si no como la transformación de un conocimiento que vincula elementos contextuales y conceptuales promoviendo su integración. Lo que debe partir de una mirada global 
Bio-grafia Escritos sobre la Biologia y su Enseñanza.

Edición Extra-Ordinaria. ISSN 2027-1034 P. p196-204

Memorias del VII Encuentro Nacional de Experiencias en la Enseñanza de la

Biología y la Educación Ambiental y II Congreso Nacional de Investigación en la

\section{Enseñanza de la Biología}

de ambiente, que logre desde un trabajo reflexivo y principalmente formativo construir la idea, que el ser humano hace parte de él, comprometiéndose a propiciar estrategias que permitan garantizar el mejoramiento del entorno en el que habita. Lo anterior se puede contrastar con el planteamiento de Meinardi (1995), en el sentido que uno de los grandes desafíos de la didáctica en la enseñanza de la Biología en los últimos años está dado en el campo de la Educación Ambiental, debido a lo difícil que resulta "poder pensar en el ambiente como un sistema formado por la interacción de los sistemas naturales y los sistemas sociales, donde ninguno de los dos existe por separado" (p. 83)

De esta manera para este trabajo es importante la formación permanente en educación ambiental de los maestros, de diversas áreas del conocimiento, partiendo de la caracterización de sus representaciones sociales sobre el concepto de ambiente y la educación ambiental. Reconociendo que la representación de ambiente que una persona 0 un grupo adopten indiscutiblemente pueden determinar sus comportamientos con respecto a esté, bien sea por acciones voluntarias o no intencionales (Sauvé, 2004). Por lo que se propone desde la formación del profesor establecer un cambio de visión sobre el ambiente en los maestros de diversas disciplinas en donde se vincule no solo a la escuela, además se logre transcender al contexto particular en el que se encuentra inmersa consiguiendo que con el saber ambiental como lo menciona Leff (2002) se asuma una posición crítica del conocimiento actual de la educación ambiental y la relación que tiene con las condiciones sociales.

Se espera establecer propuestas específicas de trabajo interdisciplinar en educación ambiental que les permita a los distintos miembros de las comunidades educativas, desde lineamientos locales vinculados a las políticas generales, construir reflexiones formativas en sus distintos ámbitos, incidiendo significativamente en la cualificación de la educación ambiental en nuestros contextos y transformando el concepto de ambiente basado en una relación ambiente igual a naturaleza hacia una visión amplia que permita entender el ambiente como un sistema del cual hacemos parte.

Se proponen proyectos de investigación en la escuela, desde la formación de maestros de diferentes áreas del conocimiento, que como agentes inmersos en las comunidades identifiquen problemáticas ambientales y logren abordarlas según su contexto particular, consiguiendo la reflexión por parte de sus comunidades frente a las posibles soluciones y contribuyendo a la construcción de un conocimiento profesional del profesor en cuanto a la educación ambiental, en el que se integren políticas de educación y se propicie la interdisciplinariedad del ambiente como posible elemento articulador del currículo escolar, aportando de esta manera a la generación de una nueva cultura ambiental. 
Memorias del VII Encuentro Nacional de Experiencias en la Enseñanza de la

Biología y la Educación Ambiental y II Congreso Nacional de Investigación en la Enseñanza de la Biología

Se requiere que en general los profesores, no solo del área de las Ciencias Naturales, expongan sus ideas sobre ambiente y educación ambiental, reflexionen y sistematicen sus prácticas pedagógicas relacionadas con la educación ambiental y formulen problemáticas ambientales desde una perspectiva interdisciplinar, integrando el conocimiento disciplinar específico a los contenidos con educación ambiental y a las necesidades contextuales (para el caso, las particulares de las localidades y las de ciudad), desarrollando conjuntamente proyectos que trasciendan el activismo.

Se pretende así, desarrollar una investigación doctoral que aporte a la formación de docentes, en aras a transformar la perspectiva reduccionista ambiente y educación ambiental en la escuela. Esto, desde un abordaje interdisciplinar, para contribuir a la construcción de una nueva cultura en comunidades educativas, la cual se incluya al ser humano como constituyente fundamental del ambiente.

\section{Bibliografía}

- Alcaldía Mayor de Bogotá. Decreto 617 de 2007. http://oab.ambientebogota.gov.co/resultado busquedas.php?AA SL Sessi on $=8 c f 97 c 692 b \& x=4083$

- Alcaldía Mayor de Bogotá. (2012). Plan de desarrollo 2012-2016, Bogotá humana.http://www.sdp.gov.co/portal/page/portal/PortalSDP/Home/Noticias/ HistoricoNoticias/PlandeDesarrollo/PLAN-DESARROLLO2012-2016.pdf

- Cohen, L. y Manion, L. (1990). Métodos de investigación educativa. Madrid.

- Constitución Política de Colombia (1991). www.banrep.gov.co/regimen/resoluciones/

- Colombia aprende. (2002). Programa de educación ambiental. Consejo Nacional Ambiental. http://aplicaciones2.colombiaaprende.edu.co/prae/contenidos/index.php

- Duarte J. y Vallejo C. (2013). Propuesta formativa en educación ambiental a partir de las representaciones sociales de ambiente y educación ambiental en el C.E. D. La Concepción. Trabajo de grado especialización - UPN. 
Bio-grafia Escritos sobre la Biologia y su Enseñanza.

Edición Extra-Ordinaria. ISSN 2027-1034 P. p196 - 204

Memorias del VII Encuentro Nacional de Experiencias en la Enseñanza de la

Biología y la Educación Ambiental y II Congreso Nacional de Investigación en la Enseñanza de la Biología

- Eschenhagen M. L. (2008) Aproximaciones al pensamiento ambiental de Enrique Leff: un desafío y una aventura que enriquece el sentido de la vida. Sección Filosofía Ambiental Sudamericana. Publicación Ocasional № 4.

- Fundación Universitaria Autónoma de Colombia. (2009). Propuesta seminario de actualización para el fortalecimiento de los proyectos ambientales escolares en el Distrito Capital. Convenio de asociación 1943.

- González, E. (2000). La transversalidad de la Educación Ambiental en el curriculum de la enseñanza básica. Reflexiones sobre la educación ambiental II. Carpeta Informativa CENEAM. Edita, organismo autónomo Parques Naturales y Ministerio de Medio Ambiente.

- Jessup M., Pulido R., León, J. (2010). Como vemos el mundo. Visiones Para el Proyecto. Representaciones Sociales de maestros rurales sobre el ambiente. Investigación desde la Calidad de Vida y el desarrollo humano Integral. p. 11-74. Bogotá D.C.

- Leff E. (2002). Saber ambiental: Sustentabilidad, racionalidad, complejidad y poder. Siglo xxi editores. México.

- Ley General de educación. Santafé de Bogotá. (1994) www.minedu.gov.co/....article-85906.html

- Meinardi, E. (2005). Estado actual del conocimiento en la didáctica de la Biología. Memorias $\mathrm{V}$ jornadas Nacionales de Enseñanza de la Biología

- Ministerio de Educación Nacional. Decreto 1743 (1994)

- Pérez, R., Contreras, Y., González, B., Martínez, J y Moreno, C. (2007). Estudio para la identificación de tendencias en educación ambiental en Bogotá. Revista Nodos y nudos. Vol. 3 № 22.

- Sauvé, L. (1994). Exploración de la diversidad de conceptos y de prácticas en la educación relativa al ambiente. Memorias Seminario Internacional. La Dimensión Ambiental y la Escuela. Serie Documentos Especiales MEN 
Bio-grafia Escritos sobre la Biologia y su Enseñanza.

Edición Extra-Ordinaria. ISSN 2027-1034 P. p196 - 204

Memorias del VII Encuentro Nacional de Experiencias en la Enseñanza de la

Biología y la Educación Ambiental y II Congreso Nacional de Investigación en la Enseñanza de la Biología

- Sauvé L. (2002). La formación continua de profesores en educación ambiental: la propuesta de EDAMA Tópicos en Educación Ambiental 4 (10), 50-62.

- Sauvé, L. (2004) Una cartografía de corrientes de educación ambiental. Cátedra de investigación Canadá en educación ambiental. Université du Québec á Montreal. (p 1-22).

- Secretaria Distrital de Planeación. (2009). Conociendo a Bogotá y sus localidades. Observatorio ambiental de Bogotá.

http://oab.ambientebogota.gov.co/resultado busquedas.php?AA SL Sessi on $=8 c f 97 c 692 b \& x=4242$

- Secretaria de Educación del Distrito. (2013). El ambiente tema de reflexión de niños y niñas en colegios Distritales. http://www.sedbogota.edu.co/index.php/noticias-secundarias/2182-elambiente,-tema-de-reflexi\%C3\%B3n--de-colegios-distritales.html

- Teixeira, V., Pereira, A., Vieira, F. (2013). Educação ambiental na escola: interdisciplinaridade e contextualização. IX Congreso internacional sobre investigación en didáctica de las ciencias.Girona 9-12.

- Universidad Pedagógica Nacional. (2013). Construcción de una ciudadanía ambiental crítica para el Distrito Capital. SED- UPN. Convenio No. 3214.

- Vasilachis, I. (2006). Estrategias de investigación Cualitativa. Barcelona, España: Gedisa Ediciones. 